\title{
End-to-End Hyperspectral-Depth Imaging with Learned Diffractive Optics
}

\author{
SEUNG-HWAN BAEK, KAIST \\ HAYATO IKOMA, Stanford University \\ DANIEL S. JEON, KAIST \\ YUQI LI, KAUST \\ WOLFGANG HEIDRICH, KAUST \\ GORDON WETZSTEIN, Stanford University
}

MIN H. KIM, KAIST

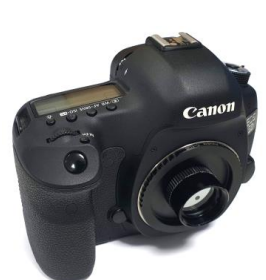

(a) Our prototype

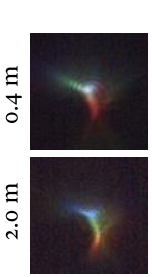

(b) HS-D PSF

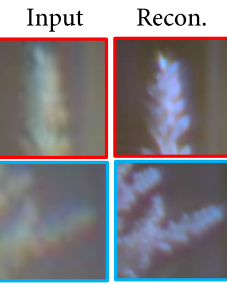

(c) Reconstructed hyperspectral image

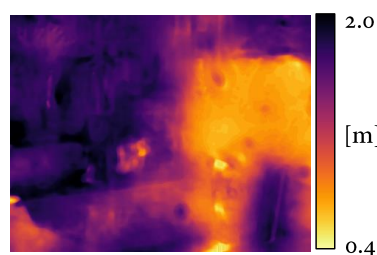

(d) Reconstructed depth

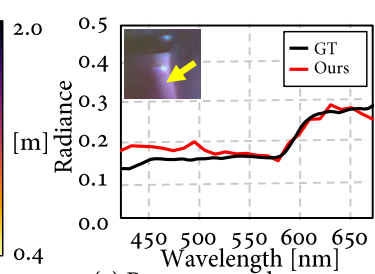

(e) Reconstructed spectrum

Fig. 1. We propose an end-to-end snapshot hyperspectral-depth imaging system with a single thin optimized diffractive optical element. (a) Our portable prototype camera is a conventional single-lens reflex camera body augmented by the optimized DOE, which creates optical point spread functions that vary with spectrum and depth. (b) Our hyperspectral-depth point spread function optically encodes the spectrum and depth information of the scene. (c) - (e) A neural network processes the snapshot input to recover both spectral and depth information of the scene.

To extend the capabilities of spectral imaging, hyperspectral and depth imaging have been combined to capture the higher-dimensional visual information. However, the form factor of the combined imaging systems increases, limiting the applicability of this new technology. In this work, we propose a monocular imaging system for simultaneously capturing hyperspectraldepth (HS-D) scene information with an optimized diffractive optical element (DOE). In the training phase, this DOE is optimized jointly with a convolutional neural network to estimate HS-D data from a snapshot input. To study natural image statistics of this high-dimensional visual data and to enable such a machine learning-based DOE training procedure, we record two HS-D datasets. One is used for end-to-end optimization in deep optical HS-D imaging, and the other is used for enhancing reconstruction performance with a real-DOE prototype. The optimized DOE is fabricated with a grayscale lithography process and inserted into a portable HS-D camera prototype, which is shown to robustly capture HS-D information. In extensive evaluations, we demonstrate that our deep optical imaging system

Authors' addresses: Seung-Hwan Baek, KAIST, School of Computing, Daejeon, South Korea, 34141; Hayato Ikoma, Stanford University, Electrical Engineering, Stanford United States, 94305-9510; Daniel S. Jeon, KAIST, School of Computing, Daejeon, South Korea, 34141; Yuqi Li, KAUST, Visual Computing Center, Thuwal, Saudi Arabia, 23955 6900; Wolfgang Heidrich, KAUST, Visual Computing Center, Thuwal, Saudi Arabia 23955-6900; Gordon Wetzstein, Stanford University, Electrical Engineering, Stanford, United States, 94305-9510; Min H. Kim, KAIST, School of Computing, Daejeon, South Korea, 34141, minhkim@kaist.ac.kr.

(C) 2020 Association for Computing Machinery.

This is the author's version of the work. It is posted here for your personal use. Not for redistribution. The definitive Version of Record was published in ACM Transactions on Graphics, https://doi.org/http://dx.doi.org/10.1145/8888888.7777777. achieves state-of-the-art results for HS-D imaging and that the optimized DOE outperforms alternative optical designs.

CCS Concepts: • Computing methodologies $\rightarrow$ Computational photography; Hyperspectral imaging; 3D imaging.

Additional Key Words and Phrases: computational imaging, deep optics

\section{ACM Reference Format:}

Seung-Hwan Baek, Hayato Ikoma, Daniel S. Jeon, Yuqi Li, Wolfgang Heidrich, Gordon Wetzstein, and Min H. Kim. 2020. End-to-End Hyperspectral-Depth Imaging with Learned Diffractive Optics. ACM Trans. Graph. 00, 0, Article 0 ( 2020), 14 pages. https://doi.org/http://dx.doi.org/10.1145/8888888.7777777

\section{INTRODUCTION}

Spectral information is crucial for a plethora of applications in the field of remote sensing [Adao et al. 2017; Näsi et al. 2015], food/agriculture [Dale et al. 2013], medical imaging [Lu and Fei 2014], and defense [Briottet et al. 2006]. Since Kim et al. [2012] introduced a combined imaging system of hyperspectral imaging and 3D imaging, many research works have sought more advanced or compact solution that can capture the higher-dimensional visual information [Feng et al. 2016; Kitahara et al. 2015; Ozawa et al. 2017; Rueda-Chacon et al. 2019; Wu et al. 2016; Zia et al. 2015]. Moreover, most of these applications require snapshot image capture and many would further benefit from the capability of capturing spectral and depth information simultaneously. 
However, traditional spectral imaging systems use spatio-spectral scanning, which makes it difficult to capture dynamic scenes. Recently proposed compressive coded aperture systems have yielded impressive results for snapshot spectral imaging [Brady 2009]. To reconstruct the spectral information of a scene from a single image, these methods must solve an inverse problem via sparse coding or machine learning [Choi et al. 2017; Jeon et al. 2019; Wagadarikar et al. 2008; Wang et al. 2019]. The reconstruction problem is severely ill-posed and not convex because the input signals are heavily compressed along the spectral dimension. Due to the high dimensionality of the problem, these existing compressive approaches are not directly able to simultaneously capture the spectral and depth information from a single image.

Simultaneous hyperspectral-and-depth imaging has been proposed, but existing systems have independently captured spectral and depth information with separate hardware, and combined the results after the fact [Feng et al. 2016; Kim et al. 2012; Wang et al. 2016; Wu et al. 2016]. These optical setups require devices with large form factors, often precluding portable applications. The key observation inspiring our approach is that depth and spectrum are closely coupled in imaging systems using diffractive optical elements, thus allowing for simultaneous capture of both modalities with the same hardware.

Here, we introduce the first snapshot approach for simultaneous hyperspectral-depth (HS-D) imaging with a single diffractive optical element (DOE). The DOE creates point spread functions on a sensor that vary with the scene depth and spectrum. We jointly optimize the surface profile of the DOE and a convolutional neural network $(\mathrm{CNN})$ in a training phase using the recent advances of end-to-end optimization of optics and image processing [Chang and Wetzstein 2019; Sitzmann et al. 2018; Sun et al. 2020b; Wu et al. 2019]. The CNN approach allows us to optimally encode the coupling between spectrum and depth, and our encoder-decoder CNN architecture further builds on this insight by using a single encoder for both dimensions of the plenoptic function but separate decoders. This approach learns a surface profile optimized for the particular application of HS-D imaging to mitigate the ill-posedness of the high-dimensional problem.

We fabricate the optimized DOE using a grayscale lithography process and mount it in front of a conventional sensor. Our prototype camera provides a small device footprint and is well suited for portable applications. During inference, this prototype captures a single RGB image from which the pre-trained $\mathrm{CNN}$ recovers both hyperspectral and depth information simultaneously.

End-to-end optimization of optics and image processing requires ground-truth training data that models natural image statistics. Unfortunately, no such dataset that would provide registered hyperspectral and depth information of realistic scenes is currently available. To mitigate this shortcoming, we built a custom multisensor rig and captured such a dataset presenting a variety of indoor scenes. To stimulate further research in this area, our dataset will be made public.

Using extensive simulations and comparisons to alternative optical designs, we demonstrate that our end-to-end approach to HS-D imaging outperforms the state of the art. Datasets, source code and optical design will be published to ensure reproducibility.
Our main contributions are:

- The first end-to-end snapshot monocular camera with learned diffractive optics that simultaneously captures a hyperspectral image and a depth map.

- The dataset of hyperspectral reflectance images and depth maps useful for HS-D imaging research.

- The optimized DOE and built a compact prototype device demonstrating its performance on both real-world indoor and outdoor scenes.

\section{RELATED WORK}

Hyperspectral imaging. Hyperspectral imaging has been extensively studied in the last decade. Scanning-based approaches capture multiple 1D spectral signals by isolating the spectral energy of each wavelength from others using a set of bandpass filters, a liquid crystal tunable filter, or a slit with dispersive optics [Brady 2009]. Compressive imaging techniques, a.k.a. coded aperture snapshot spectral imagers (CASSI), enable single-shot capture of hyperspectral images [Jeon et al. 2016; Johnson et al. 2007; Wagadarikar et al. 2008]. Recent approaches have demonstrated the potential of estimating hyperspectral images from spectrally varying point spread functions (PSFs) [Baek et al. 2017; Jeon et al. 2019] in a compact configuration that make use of edge information instead of using the modulated aperture mask. Our approach extends the capabilities of these spectrum-from-PSF methods by taking a first step towards snapshot imaging of higher-dimensional visual data: the spectrum as well as depth.

Hyperspectral-depth imaging. HS-D imaging has been explored by combining different imaging systems for spectrum and depth. For example, passive stereo [Ito et al. 2016; Wu et al. 2016; Zia et al. 2015] and active stereo [Kim et al. 2012; Kitahara et al. 2015; Ozawa et al. 2017] have been employed in conjunction with spectral cameras [Kim et al. 2012; Ozawa et al. 2017; Wu et al. 2016; Zia et al. 2015] and spectral light sources [Ito et al. 2016; Kitahara et al. 2015]. These approaches use two different imaging modalities for spectral and depth information, significantly increasing the device form factors and, in many cases, making it difficult to match stereo features across different spectral bands. CASSI systems have also been combined with light-field or time-of-flight (TOF) imaging to achieve snapshot monocular imaging [Feng et al. 2016; RuedaChacon et al. 2019], but these systems use custom-build optical coding strategies which are restricted to indoor scenes only. To date, these systems have only been demonstrated on an optical table with a large form factor, limiting its applications. Furthermore, parallax and related alignment problems across modalities can negatively affect the reconstruction results. Thus, existing HS-D imaging methods are inapplicable for use in portable applications. In contrast, we demonstrate a portable prototype HS-D imaging system with a single thin optimized diffractive optical element operating in a fully passive way without additional registration of depth and spectrum.

Deep optics. The idea of jointly optimizing optical elements with differentiable reconstruction algorithms has recently been explored for various applications, including color filter design [Chakrabarti 


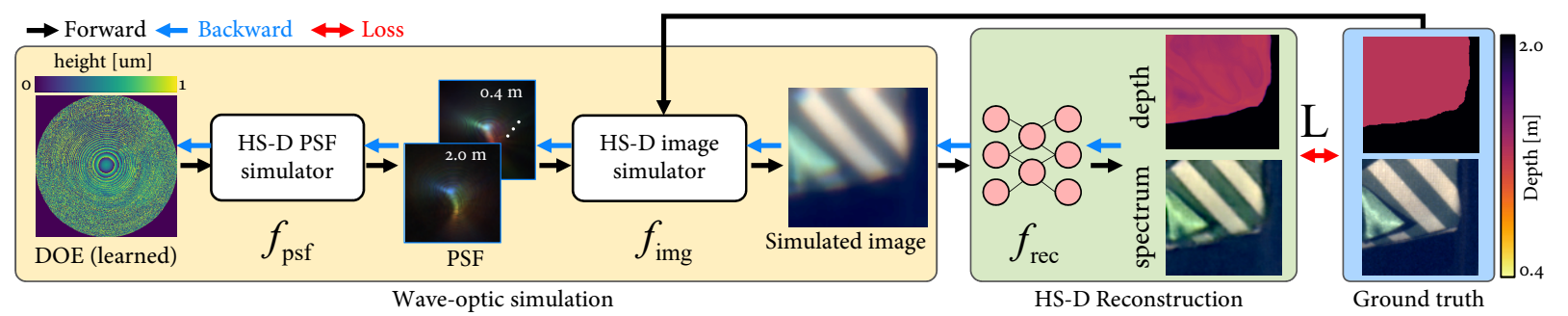

Fig. 2. Overview. We jointly optimize the height field of a diffractive optical element and a deep neural network for snapshot hyperspectral-depth imaging. In a forward pass, a point spread function is simulated for every spectral slice and depth via a wave optics-based PSF simulator. This simulator uses the generated PSFs to compute a single 2D RGB image measurement corresponding to some incident ground truth hyperspectral radiance image and depth map. The neural network tries to reconstruct the hyperspectral-depth data from the simulated sensor image and a loss function measures the difference from the ground truth. Both the image formation model and neural network are differentiable, which allows us to optimize the DOE surface profile and the network parameters jointly through error backpropagation.

2016], spectral imaging [Wang et al. 2019], superresolution localization microscopy [Nehme et al. 2019], super-resolution SPAD imaging [Sun et al. 2020b], depth estimation [Chang and Wetzstein 2019; Haim et al. 2018; Wu et al. 2019], extended depth of field and superresolution imaging [Sitzmann et al. 2018], HDR imaging [Metzler et al. 2020; Sun et al. 2020a], and image classification [Chang et al 2018]. Based on this paradigm, we train a DOE for HS-D imaging while learning a reconstruction network. Our approach is the first to propose and successfully demonstrate end-to-end optimization of a single DOE and a CNN for snapshot hyperspectral and also hyperspectral-depth imaging.

\section{OVERVIEW}

The goal of our computational imaging system is to simultaneously capture optically aligned hyperspectral and depth images of a scene in a single exposure. Figure 2 shows an overview of the proposed end-to-end training procedure. Here, we first simulate point spread functions (PSFs) for discrete samples of depth and spectrum given a height field of the DOE. A dataset of hyperspectral-depth images, which we captured with a custom setup, is used to simulate captured RGB images by applying the simulated PSFs. The CNN takes a (simulated or real) captured image as input and reconstructs both a hyperspectral image and a depth map in the range of 420-660 nm with $10 \mathrm{~nm}$ intervals as well as a depth map with a target range of $0.4-2.0 \mathrm{~m}$. This procedure is implemented in a fully differentiable manner to enable error backpropagation into the DOE height field as well as the reconstruction algorithm via automatic differentiation.

\section{SPECTRAL-DEPTH IMAGE FORMATION}

Our image formation model builds on a differentiable wave optics simulator [Goodman 2005]. PSFs are used to simulate a captured image by convolving the PSFs with hyperspectral-depth data:

$$
J_{c \in\{R, G, B\}}=f_{\text {img }}\left(P_{\lambda, z}, I_{\lambda}, Z\right),
$$

where $J_{c \in\{R, G, B\}}$ is the simulated captured image, $I_{\lambda}$ is the groundtruth hyperspectral image, $Z$ is the ground truth depth map, and $f_{\text {img }}$ is the differentiable image simulator. We describe details of each part in the following.

\subsection{Point spread function}

We first simulate PSFs for the given DOE height map $h$ for target spectrum and depth candidates:

$$
P_{\lambda, z}=f_{\mathrm{psf}}(h),
$$

where $P_{\lambda, z}$ is the PSF for wavelength $\lambda$ and depth $z$, and $f_{\mathrm{psf}}$ is the differentiable PSF simulator. Suppose we have a scene point at depth $z$. The wave field of wavelength $\lambda$ originating from the scene point can be modeled as a spherical wave $U_{\lambda, z}$ at the location just before the DOE with Fresnel approximation, assuming $\lambda \ll z: U_{\lambda, z}^{(1)}=$ $\exp \left[i \frac{2 \pi}{\lambda} \frac{x^{\prime 2}+y^{\prime 2}}{z}\right]$. Here, $\left(x^{\prime}, y^{\prime}\right)$ is the spatial location on the DOE plane as shown in Figure 3. The wave field then passes through the camera aperture and the DOE resulting in changes of the amplitude and phase: $U_{\lambda, z}^{(2)}=A\left(x^{\prime}, y^{\prime}\right) \cdot \exp \left[i \frac{2 \pi}{\lambda}\left(\frac{x^{\prime 2}+y^{\prime 2}}{z}+\left(\eta_{\lambda}-1\right) h\left(x^{\prime}, y^{\prime}\right)\right)\right]$, where $A$ is the amplitude aperture function, which is 0 for the blocked region and 1 elsewhere, and $\eta_{\lambda}$ is the refractive index of the DOE material for wavelength $\lambda$. Next, the wave field propagates a distance $f$ to the sensor, resulting in the point spread function $P_{\lambda, z}$, which is the squared magnitude of the complex wave field at the sensor plane:

$$
\begin{array}{r}
P_{\lambda, z}=\mid \mathcal{F}\left\{A ( x ^ { \prime } , y ^ { \prime } ) \cdot \operatorname { e x p } \left[i \frac { 2 \pi } { \lambda } \left(\frac{x^{\prime 2}+y^{\prime 2}}{z}+\left(\eta_{\lambda}-1\right) h\left(x^{\prime}, y^{\prime}\right)\right.\right.\right. \\
\left.\left.\left.+\frac{1}{2 f}\left(x^{\prime 2}+y^{\prime 2}\right)\right)\right]\right\}\left.\right|^{2} .
\end{array}
$$

We computed the PSF for a discrete set of wavelengths $\lambda \in \Lambda$ and depths $z \in Z$.

In contrast to existing approaches for PSF-based imaging [Chang and Wetzstein 2019; Sitzmann et al. 2018; Wu et al. 2019], which use only three wavelengths (typically, 450, 550 and $650 \mathrm{~nm}$ ) for simulating trichromatic-channel PSFs, we perform dense spectral sampling of 25 spectral channels from 420 to $660 \mathrm{~nm}$ in $10 \mathrm{~nm}$ intervals. This is essential not only for estimating a hyperspectral image from the spectral cue of PSFs, but also for accurately simulating the image formation model. For depth, we sample seven values from 0.4 to $2.0 \mathrm{~m}$ linearly in disparity. Refer to Section 3 in the supplemental document for more details on hyperspectral-depth PSF. 


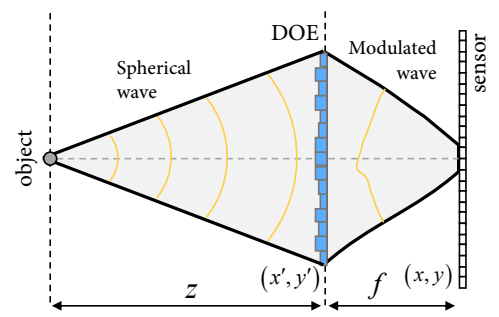

Fig. 3. Light propagation. Suppose an object is placed at distance $z$ from the camera, and the reflected light from the object arrives at the diffractive optical element as a spherical wave. The DOE with optimized height profile modulates the phase of the incident spherical wave. The camera sensor then captures the propagated wave field.

\subsection{Image formation}

Given the PSF $P_{\lambda, z}$, the all-in-focus hyperspectral image and the all-in-focus depth map, we can simulate an RGB sensor image. For PSF-based image formation, we make use of a layered scene representation [Chang and Wetzstein 2019; Wu et al. 2019]. We treat the scene as a set of multiple segmentation layers at different depth levels. We simulate the hyperspectral image captured by the sensor by convolving each layer with the spectral PSFs for that layer. Next, we apply the camera response function $\left(\Omega_{c \in\{R, G, B\}, \lambda \in \Lambda}\right)$ of the sensor to convert the captured spectral image to RGB. In summary, the captured RGB image $J_{c \in\{R, G, B\}}$ is formulated as follows:

$$
\begin{aligned}
J_{c \in\{R, G, B\}} & =f_{\text {img }}\left(P_{\lambda, z}, I_{\lambda}, Z\right) \\
& =\sum_{\lambda \in \Lambda} \Omega_{c, \lambda} \sum_{z \in Z} M_{z} \odot\left(I_{\lambda} \otimes P_{\lambda, z}\right)+n,
\end{aligned}
$$

where $\odot$ is an element-wise product operator, $\otimes$ is a convolution operator, and $M_{z}$ is the binary mask for each depth layer $z$, where the value is one if the pixel depth is at $z$, and zero otherwise. We further convert the Boolean mask $M_{z}$ to a real-values one by applying a Gaussian filter. This improves the handling of object boundaries as an approximation of the physical occlusion effect [Chang and Wetzstein 2019; Wu et al. 2019]. To account for noise, we apply Gaussian noise $n$ with a standard deviation $\sim 4 \cdot 10^{-4}$.

Contrary to previous PSF-based depth imaging [Chang and Wetzstein 2019; Sitzmann et al. 2018; Wu et al. 2019], our hyperspectraldepth image formation accounts for more dense spectral and depth samples (25 in spectrum and 7 in depth), more accurately describing continuous image formation in real world.

\subsection{Analysis of spectrum and depth dependency}

As described by Equation (3) and seen in Figure 3, the PSF generated by a DOE in an imaging system depends on the spectral wavelength $\lambda$ and depth $z$ of the object. See the phase term in Equation (3) below:

$$
\frac{2 \pi}{\lambda} \frac{x^{\prime 2}+y^{\prime 2}}{z}+\frac{2 \pi}{\lambda}\left(\eta_{\lambda}-1\right) h\left(x^{\prime}, y^{\prime}\right)+\frac{2 \pi}{\lambda} \frac{x^{\prime 2}+y^{\prime 2}}{2 f} .
$$

The phase term consists of three terms: (a) the light propagation from the object to the DOE, (b) the phase delay by the DOE, and (c) the light propagation from the DOE to the sensor. The first term is inversely proportional to both wavelength $\lambda$ and depth $z$, the second term is proportional to the refractive index $\eta_{\lambda}$ of the DOE material and inversely proportional to $\lambda$, and the last term is also

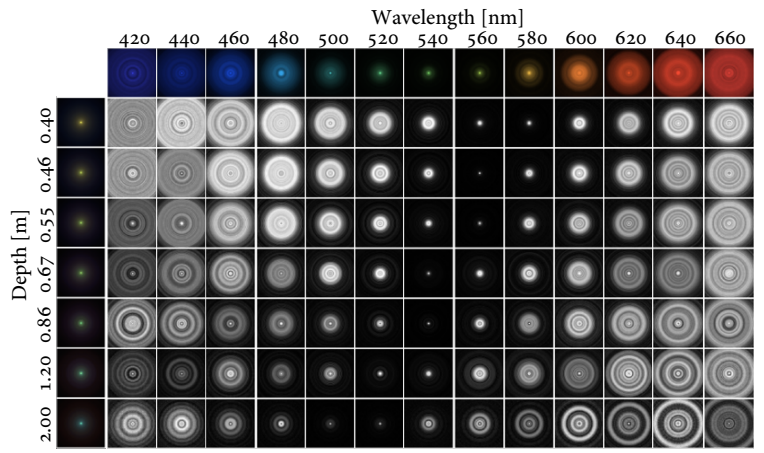

Fig. 4. Spectrum and depth dependency of the PSF. The captured phase is related to the spectrum and depth of the object. The $y$-axis is represented as the linear scale in the inverse depth. The $x$-axis is shown in 20-nm intervals.

inversely proportional to $\lambda$ and the focal length $f$ of the DOE. One of the key insights of our work is that the DOE phase affects both the spectrum and depth of a recorded scene. Therefore, we can in principle reconstruct these two pieces of information from a single image.

Figure 4 presents a simulation example for the PSF of a Fresnel DOE by varying the wavelength of light and object depth. We observe the predicted spectrum-depth ambiguity along the diagonal lines, e.g., the PSF of low wavelength at a far distance is similar to the PSF of long wavelength at a near distance. A traditional PSF engineering approach would likely aim at directly optimizing some property of the PSFs to mitigate this ambiguity. Note that we do not follow this strategy, but rather optimize the DOE in an end-to-end manner together with the reconstruction network. This approach allows us to place the loss directly on the estimated HS-D data, rather than on some proxy metric computed for the PSF. Our approach, however, has the drawbacks that the resulting PSFs are not necessarily interpretable and they depend on the loss function, the reconstruction algorithm, and also the dataset used for training.

\section{END-TO-END HS-D RECONSTRUCTION}

Suppose that we install a DOE in front of a bare sensor of a conventional DSLR camera as shown in Figure 1(a). Our goal is to reconstruct a hyperspectral image $\hat{I}_{\lambda}(x, y)$ and a depth map $\hat{Z}(x, y)$ from a captured RGB image $J_{c \in\{R, G, B\}}(x, y)$ by exploiting the PSF characteristics for spectrum and depth:

$$
\hat{Z}, \hat{I}_{\lambda}=f_{\text {rec }}\left(J_{c \in\{R, G, B\}}\right),
$$

where $f_{\text {rec }}$ is a reconstruction algorithm. This reconstruction problem is challenging for several reasons. First, while the input RGB image has only three spectral channels, we wish to reconstruct from this input both a hyperspectral image with many more channels (e.g., 25), as well as a depth map. Second, the ambiguity between spectrum and depth in the PSF further complicates the reconstruction. In order to overcome these challenges, we use a deep neural network as an effective reconstruction algorithm.

\subsection{Network architecture}

Our HS-D PSF and image simulation of 25 wavelengths at 7 depth planes highly demands GPU memory. Therefore, we designed our network simply by modifying a U-Net [Ronneberger et al. 2015] 


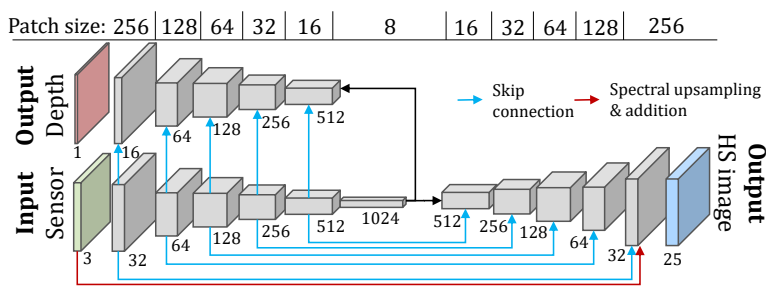

Fig. 5. Reconstruction network. The network takes as input a single RGB image patch. Using a shared encoder and two different decoders, each with skip connections from the encoder, the network estimates both a hyperspectral image with 25 channels and a depth map. The numbers below each feature denotes the number of feature channels and the patch size is described at the top.

with two decoders: one for depth and the other for the HS image (Figure 5). Different from the original U-Net, we apply residual learning by adding the initial spectral tensor via spectral upsampling to the output HS image. Images are always processed as patches, both during both training and inference. We typically use patches with a resolution of $256 \times 256$. We target a spectral range of the hyperspectral images from 420 to $660 \mathrm{~nm}$ in $10 \mathrm{~nm}$ intervals.

Spectral upsampling. The number of the output spectral channels is larger than that of the input spectral channels in our reconstruction problem: from 3 to 25. Since we reconstruct the solution using gradient descent, our reconstruction method requires careful initialization. We first approximate the initial energy in the spectral tensor by distributing the energy of the three color channels to the hyperspectral channels according to the camera response functions. For example, the energy of the red channel is distributed into the range of the visible spectral bands according to the spectral response weights of the red channel in the camera. We calculate the spectral sensitivities of each wavelength by accounting for three color filter responses of the camera: $I_{\lambda}=\Sigma_{c} w(\lambda, c) J_{c}$, where $w(\lambda, c)=\Omega(\lambda, c) /\left\{\sum_{c^{\prime}} \Omega\left(\lambda, c^{\prime}\right) \sum_{\lambda^{\prime}} \Omega\left(\lambda^{\prime}, c\right)\right\}$ and $c, c^{\prime} \in\{r, g, b\}$. This initial approximation is added to the output of the hyperspectral decoder, realizing residual learning in the spectral dimension.

\subsection{Loss function}

During training, we use the index of depth as a representation for depth, which is linearly sampled in disparity space (i.e., inverse meters). To define a loss function $\mathcal{L}$, we enforce the mean absolute error (MAE) for the depth and the hyperspectral image with total variation (TV) regularization on depth:

$$
\mathcal{L}=\alpha \frac{1}{N}\left\|\hat{I}_{\lambda}-I_{\lambda}\right\|_{1}+\beta \frac{1}{M}\|\hat{Z}-Z\|_{1}+\gamma \frac{1}{M}\|\nabla Z\|_{1},
$$

where $N$ and $M$ are the number of total pixels in the hyperspectral image and the depth map, respectively, and $\nabla$ is the spatial gradient operator. The corresponding weights are given by $\alpha, \beta$, and $\gamma$, respectively.

\subsection{DOE initialization}

Jointly optimizing a DOE and a CNN for hyperspectral-depth imaging is a challenging, non-convex inverse problem that aims at simultaneously solving multiple traditional problems, including phase retrieval, spectral super-resolution, monocular depth estimation, and deconvolution. The non-convex nature of this problem makes it crucial to find a good initialization of the optimization parameters. In particular, the initialization of the DOE has been shown to be important and is specific to a target application. For hyperspectraldepth imaging, we therefore seek to find a proper initialization of the DOE through a Fisher-information-based optimization to obtain the initial DOE height field [Shechtman et al. 2014].

Since the Fisher information matrix for the general hyperspectral depth imaging problem is too large to evaluate, we consider a simpler subproblem where we estimate the location and wavelength of a monochromatic point-source emitter from its single RGB image $\left(J_{c}\right)$. Its Fisher information matrix $\mathcal{I}$ then describes the sensitivity of the observed PSF to the spatial emitter positions $\left(p_{x}, p_{y}, p_{z}\right)$ and wavelengths $\left(p_{\lambda}\right)$. When the brightness of the point source is known, the Fisher information matrix under the Gaussian noise model is given as:

$$
\mathcal{I}_{i j}(\delta)=\sum_{c, k} \frac{1}{\sigma^{2}} \frac{\partial J_{c}(k ; \delta, h)}{\partial \delta_{i}} \frac{\partial J_{c}(k ; \delta, h)}{\partial \delta_{j}},
$$

where $\delta=\left\{p_{x}, p_{y}, p_{z}, p_{\lambda}\right\}, \sigma$ is the standard deviation of the Gaussian noise, $k$ is the pixel index, and $h$ is the DOE height field. While the Fisher information depends on the position and wavelength of the point-source emitter, we aim to find a DOE height field that provides high Fisher information for all sources in our design space. To achieve this, we optimize the height of the DOE by minimizing the mean of the $A$-optimality of the Fisher information matrix over a set of monochromatic point sources located on the optical axis:

$$
\underset{h}{\operatorname{minimize}} \frac{1}{N} \sum_{p_{\lambda} \in \Lambda} \sum_{p_{z} \in \mathbf{z}} \mathcal{A}\left(p_{z}, p_{\lambda} ; h\right),
$$

where $\mathcal{A}$ is the $A$-optimality, which is the trace of the inverse of the Fisher information matrix $\mathcal{I}$. The design space of the imaging system is characterized by the set of wavelengths $\Lambda$ and the set of the depth layers $\mathbf{z}$ where the point sources are placed. Since Equation (9) is not a convex problem, we solve it based on stochastic gradient descent optimization, using the Adam optimizer. This optimization itself requires an initialization, for which we choose a conventional Fresnel DOE lens pattern. We set the brightness of the point source so as to ensure the maximum intensity of the captured PSFs of a Fresnel lens is 0.8 of the maximum intensity of the image.

Phase vs. height map. Existing deep optics approaches [Chang and Wetzstein 2019; Sitzmann et al. 2018] directly optimize the height field of the DOE, but it imposes a phase wrapping problem when optimizing the DOE parameters. We therefore optimize the unwrapped phase shift $\phi$, which is directly related to the DOE height $h$ for a reference wavelength of $550 \mathrm{~nm}: h=\frac{\lambda}{2 \pi} \frac{\phi_{\text {wrap }}}{\left(\eta_{\lambda}-1\right)}$, where $\phi_{\text {wrap }}$ is the wrapped phase in the range of $[0,2 \pi]$. Contrary to the height field, the unwrapped phase has no range restriction and is later wrapped in a post-processing stage [Damberg et al. 2016]. The phase can be mapped one-to-one to a height field geometry by taking the refractive index of the DOE material into account. 


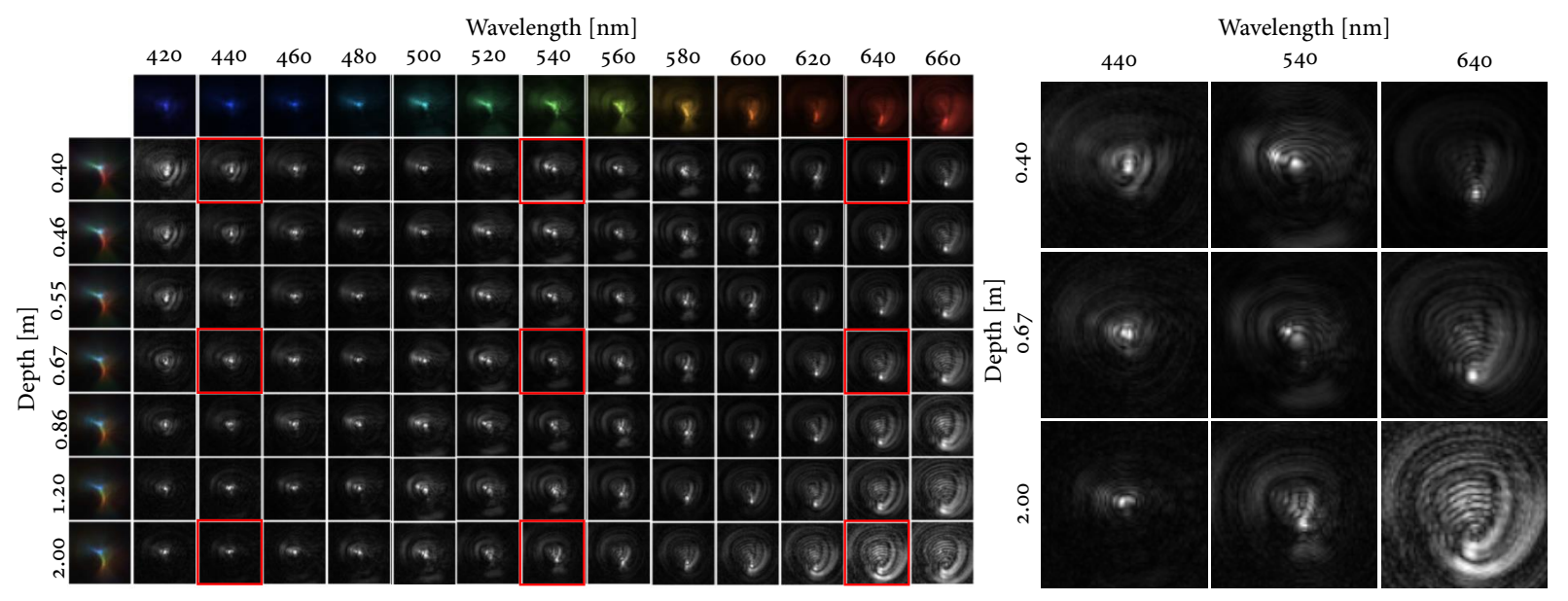

Fig. 6. Optimized PSF for HS-D imaging. Each column and row corresponds to a spectral band and a depth candidate. We visualize the PSF slices for each spectrum and depth by normalizing it. The top row shows the sum of PSF slices at different depth candidates for a wavelength while the leftmost column shows RGB visualization of the spectral PSFs at each depth level. Our optimized PSF shows spectral/depth variation that we can exploit for HS-D reconstruction.

\subsection{Training}

Since the CNN is differentiable, our full optimization of optics and the reconstruction algorithm is formulated in an end-to-end manner, resulting in the height map of the learned DOE and the parameters of the HS-D reconstruction network:

$$
\underset{h, \theta}{\operatorname{minimize}} \mathcal{L}\left(\left\{\hat{Z}(h, \theta), \hat{I}_{\lambda}(h, \theta)\right\},\left\{Z, I_{\lambda}\right\}\right),
$$

where $h$ is the DOE height, $\theta$ is a set of network parameters, and $\mathcal{L}$ is the loss between reconstruction and ground truth. The Fisherinformation-based DOE initializer optimizes the PSF for a single monochromatic point light source for the sake of simplicity. Our end-to-end method then further optimizes it to be robust to realworld scenes of complex texture and extended multiple objects, resulting in focused PSFs with depth and spectral variation.

Figure 6 presents the optimized PSFs after end-to-end optimization. It visualizes the PSF slices for each spectrum and depth. We initialized with the aforementioned Fisher-information-based DOE patterns. Our final optimized PSF contains anisotropic structures with spectral and depth variation that the reconstruction network exploits to reconstruct HS-D images with high accuracy.

Network training. Our end-to-end HS-D reconstruction is implemented in Pytorch and uses the Adam optimizer for training. Refer to Section 1 in the supplemental document for details of the network architecture. The total number of network parameters is $39,484,378$. The learning rates for the DOE phase and network weights are set as $10^{-4}$. We decay the learning rates differently for the DOE and network by 0.1 per 10 epochs and 0.1 per 20 epochs, respectively, following [Wu et al. 2019]. Once the DOE shape is converged, we fix the DOE and keep training the network for training efficiency. End-to-end training took 12 epochs in about 48 hours, after which the reconstruction part was trained for an additional 30 epochs, also taking 48 hours. The training was done using a workstation equipped with a $3.40 \mathrm{GHz}$ Intel i7-3770 CPU, $32 \mathrm{~GB}$ of main memory, and an NVIDIA Titan Xp GPU with 12 GB memory. For testing, it took about 1.45 seconds to reconstruct a hyperspectral-depth image with the resolution of $1412 \times 2120$.

\subsection{Training datasets}

In this work, we recorded two different datasets for (a) end-to-end optimization of the DOE design and the reconstruction network and (b) refining the reconstruction network for the real-DOE prototype.

$H S$ - $D$ dataset. For end-to-end optimization, we need a dataset of spectral reflectance and objects at different distances, where the images should represent natural spectral statistics and be captured without blur. Note that to train the reconstruction network, each object is synthetically blurred by the known PSFs for the appropriate distances and wavelengths. However, there is no spectrum-depth image dataset suitable for this task, while different modal image datasets of spectrum [Chakrabarti and Zickler 2011; Choi et al. 2017; Yasuma et al. 2010] and depth [Silberman et al. 2012; Song et al. 2015] exist. We therefore create an HS-D dataset of 18 scenes consisting of 73 different objects in addition to a ColorChecker and a Spectralon. Our dataset consists of optically aligned pairs of a hyperspectral image and a depth map (see Figures 7(a) and (b)). Hyperspectral images are captured in the spectral range from $420 \mathrm{~nm}$ to $680 \mathrm{~nm}$ in $10 \mathrm{~nm}$ intervals and then converted to reflectance maps through radiometric calibration so that the spectral information of the scenes can later be augmented to radiance images by multiplying them with different standard illuminants. We capture the depth information of individual objects using the structured light method [Lanman and Taubin 2009]. The depth of captured objects varies within a range of $\sim 0.4 \mathrm{~m}$ to $2.0 \mathrm{~m}$. For the layered scene representation, we quantize continuous depth values into seven depth levels sampled linearly in disparity space. The spatial resolutions of the HS image and the depth map are both $2704 \times 3376$. We also provide a background mask for each scene, which is useful for extracting valid training patches.

We divide the 18 scenes into 13 scenes for training and 5 for testing. We then collect $256 \times 256$-sized HS-D patches. Background dominant patches having invalid depth values or too low intensity are excluded for both training and testing. We also ensured that 


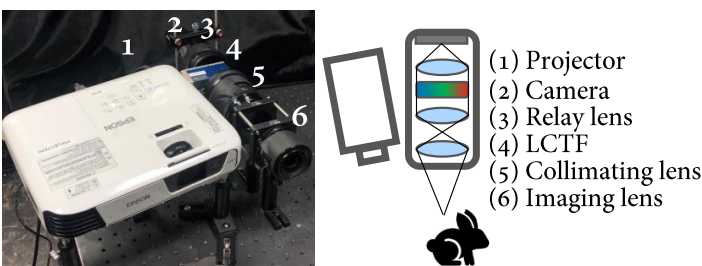

(a) Acquisition setup for HS-D dataset, schematic diagram

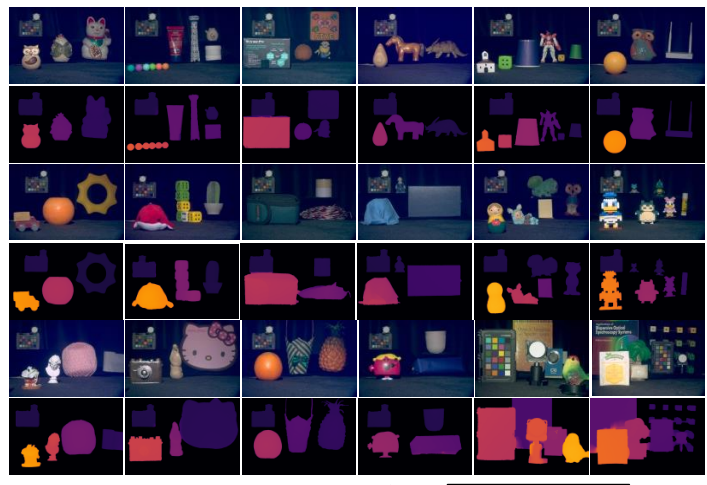

(b) Our HS-D dataset

Depth 0.4

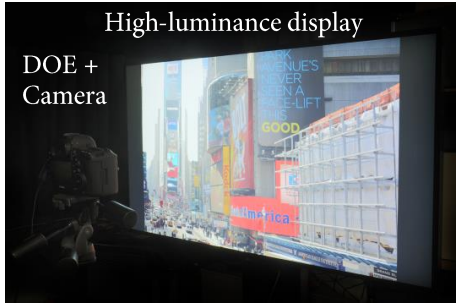

(c) Acquisition setup for refinement

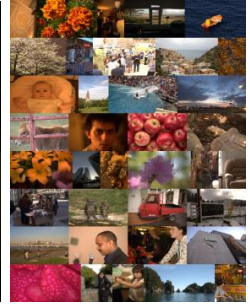

(d) thumbnail
Fig. 7. HS-D datasets. (a) A hyperspectral-depth imaging setup that consists of a projector for structured light scanning and an LCTF-based hyperspectral imager. (b) This dataset consists of pairs of a hyperspectral reflectance image and a depth map. (c) Another acquisition setup to record a pair of HS-D information of natural images (Adobe FiveK), specifically used to mitigate the artifacts by diffraction inefficiency in the real-DOE prototype.

only one of the 13 training scenes includes a ColorChecker in a set of training minibatches in order to avoid overfitting to this target. Also, a few test scenes include the ColorChecker for the sake of evaluation. Refer to Section 2 in the supplemental document for details on data augmentation.

Refinement dataset. To mitigate the performance gap between the synthetic optimization and the real prototype camera, we recorded another dataset. See Section 6.3 for more details. Once we fabricated the DOE design, we captured 400 natural images (selected from the MIT-Adobe FiveK dataset [Bychkovsky et al. 2011]) displayed on a high-luminance 55-inch display (LG signage 55XS2B, peak luminance: $\left.2,500 \mathrm{~cd} / \mathrm{m}^{2}\right)$ using the real-DOE camera, at 7 different depths from 0.4 to $2.0 \mathrm{~m}$, resulting in 2,800 images in total. See Figures 7(c). At the same time, we captured hyperspectral images using a custombuilt hyperspectral camera (a machine vision camera equipped with a liquid-crystal bandpass filter in front of the objective lens.). These
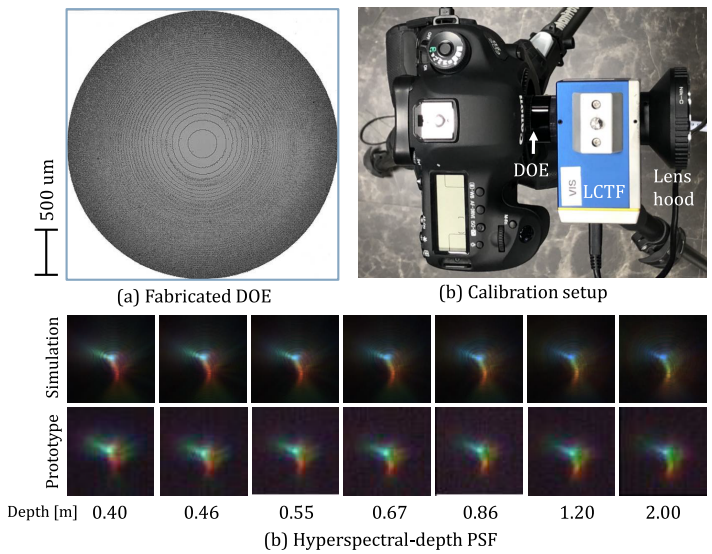

Fig. 8. DOE fabrication and PSF calibration. (a) Our fabricated DOE height profile captured by a microscope. (b) PSF calibration setup with the fabricated DOE and the LCTF. (c) RGB visualization of the HS-D PSFs of simulated PSF and fabricated PSF.

hyperspectral images are registered to the images taken by the prototype camera by deriving a set of homography matrices estimated by the checkerboard-calibration target.

\section{REAL PROTOTYPE}

\subsection{HS-D camera prototype}

To build our HS-D camera prototype, we employed a Canon 5D Mark III camera with a pixel pitch of $6.22 \mu \mathrm{m}$ and a resolution of $3840 \times 5760$ pixels. The designed distance from the DOE to the sensor is set to $50 \mathrm{~mm}$. We fabricate the optimized DOE as described below. It is mounted to a C-mount tube, which is attached to the camera body with an EOS-C adapter. We made an additional C-mount extender with a 3D printer to place the DOE at the exact distance from the sensor. Refer to Section 4 in the supplemental document for calibration details.

\subsection{DOE fabrication}

The DOE height map is parameterized as a bitmap with a resolution of $375 \times 375$ features and a pixel pitch of $8 \mu \mathrm{m}$, resulting in a DOE aperture of $3 \mathrm{~mm}$. Note that, for fabrication, we upsample the DOE height field to a resolution of $3000 \times 3000$ of $1 \mu \mathrm{m}$ pixel pitch with nearest neighbor interpolation to match with the simulation process, and quantize the height range to 62 levels $(21.5 \mathrm{~nm} /$ level).

The diffractive optical element is fabricated through soft lithography [Xia and Whitesides 1998]. A master mold is made with positive photoresist (AZ-1512, MicroChemicals) spun on a titanium-coated glass substrate. The pattern is written by a direct-write gray-scale photolithography machine (MicroWriter ML3, Durham Magneto Optics Ltd) and developed with a MF-319 developer (Microposit). After the development, polydimethylsiloxane (PDMS, SYLGARD 184 , Dow) is cast and cured at room temperature with the master mold to form another mold. This PDMS mold is used to transfer the pattern to a $3 \mathrm{~mm}$-thick float glass substrate (30-773, Edmund Optics).

The glass substrate is preprocessed to form a circular aperture with the layers of chromium and gold through a lift-off process. A 
drop of UV-curable resin (NOA61, Norland Products, its refractive index at $546.1 \mathrm{~nm}$ is 1.5634 ) is then sandwiched between the glass substrate and the PDMS mold, and is exposed to a mercury-vapor lamp to cure the resin. After the PDMS mold is peeled off, the pattern is replicated on the NOA61-resin layer which acts as a DOE. As the fabrication accuracy of the DOEs cannot be directly measured due to their microscale patterns, the accuracy of the fabrication system was indirectly measured on 15 reference holes which are designed to have different depths over $2 \mu \mathrm{m}$. The depths of the fabricated reference holes were measured with a profilometer (KLA Tencor Alpha Step D-500). The RMSE of the 15 sample points was $173.2 \mathrm{~nm}$, and the estimated quantization scale was $20.5 \mathrm{~nm} /$ level The remaining area of the glass substrate is covered by a chrome aperture mask of the same diameter $(3 \mathrm{~mm})$ placed on the same side where the DOE is printed.

\subsection{Refinement of the reconstruction network}

After we built the prototype with the fabricated DOE and calibrated the PSF of the prototype, we found that low diffraction efficiency of the real DOE causes a long tail of PSF with low levels of intensity (similar to noise), forming a very large convolution kernel. To meet the requirement of memory footprint in GPU, we excluded the noisy long tail from our real PSF model. It results in common hazy artifacts also observed in previous works of DOE engineering works [Jeon et al. 2019; Sitzmann et al. 2018].

We extended a recent approach [Peng et al. 2019] that mitigates the hazy artifacts from DOE images at a single depth level. Instead, we captured a set of natural spectral images and the prototype camera input at different distance levels, yielding the real-DOE training dataset (shown in Figures 7(c)).

By doing so, we can refine the parameters of our reconstruction network using the real-DOE training dataset. As each training patch consists of a constant depth value, we perform patch-wise reconstruction at test time and reconstructs the final output via the mean of overlapping patches.

This additional refinement compensates the physical gap between the synthetically optimized DOE and the fabricated DOE, which causes low diffraction efficiency. We found that this additional step can improve the accuracy of spectral and depth images captured by the real-DOE prototype.

\section{RESULTS}

We conducted quantitative and qualitative evaluations of our method on our HS-D dataset in simulation and real scenes captured by our prototype. In simulation, we used a test of 145 HS-D images including five different scenes of reflectance illuminated by 29 different CIE illuminants. For the real scenes, we captured indoor and outdoor scenes with our real prototype under conventional LED light and sunlight, respectively. We measured spectrum and depth information in the real scene with a spectroradiometer (SpectraScan 655) and a laser distance meter (Bosch GLM 80) to compare our results to reference.

For spectral accuracy per pixel, the average peak signal-to-noise ratio (PSNR) and structural similarity (SSIM) are calculated. For depth accuracy per pixel, the root mean squared error (RMSE) [m] and the mean absolute error (MAE) [m] are used. Note that these depth accuracy metrics are averaged out from valid pixel measurements only, following conventional per-pixel depth estimation criteria [Uhrig et al. 2017].

Comparison to other HS-D imaging. We compare our HS-D imaging method with a state-of-the-art HS-D imaging method [Feng et al. 2016] in simulation (Figure 9 and Table 1). The existing HS-D system is built by combining two imaging modalities: compressive hyperspectral imaging and light-field imaging. The baseline for spectral imaging is the same as CASSI, which consists of a coded aperture mask, relay lenses, and a prism. An additional microlens array is attached in front of the CASSI module to acquire depth information from the light field. This system requires hyperspectral and depth information from sparse input signals, which are heavily compressed in the angular-spectral space. Their results thus suffer from severe noise and reconstruction artifacts in the reconstructed spectrum and depth information, as shown in Figure 9(b). In contrast, our HS-D system is rather simple, comprising a single DOE and imaging sensor, but our results outperform the state-of-the-art method in terms of spectrum and depth accuracy.

Comparison with other DOE patterns. We evaluate the accuracy of our hyperspectral-depth imaging with our end-to-end DOE, compared with different DOE patterns. To estimate spectrum and depth information, the same network architecture (described in Section 5) was trained with four different DOEs: the traditional Fresnel-lens DOE, the spiral-shaped DOE [Jeon et al. 2019], Fisher-informationbased DOE [Shechtman et al. 2014], and our DOE learned by endto-end (E2E) optimization.

Figure 10 and Table 2 compare the average accuracy of a test set of the 145 HS-D images, synthetically compared against groundtruth data. For these three fixed DOEs, the trained neural network reconstructs spectrum and depth information well by exploiting the natural statistics of the training HS-D data. The Fresnel-lens DOE results show good accuracy in estimating depth information

\begin{tabular}{|c|c|c|c|}
\hline \multicolumn{2}{|c|}{ HS-D imaging } & Feng et al. & Ours \\
\hline 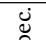 & PSNR [dB] & 23.62 & 29.31 \\
\hline के & SSIM & 0.76 & 0.81 \\
\hline F & RMSE [m] & 0.57 & 0.20 \\
\hline $\overrightarrow{\breve{~}}$ & MAE [m] & 0.30 & 0.12 \\
\hline
\end{tabular}

Table 1. Comparison of spectral and depth accuracy to a light-field-based HS-D imaging [Feng et al. 2016] in simulation. The average spectral and depth accuracy of our method are significantly better than those of the light-field-based method.

\begin{tabular}{|c|c|c|c|c|c|}
\hline \multicolumn{2}{|r|}{$\mathrm{DOE}$} & Fresnel & Spiral & Fisher & E2E (ours) \\
\hline \multirow{2}{*}{$\begin{array}{l}\text { ठั. } \\
\text { के }\end{array}$} & PSNR [dB] & 27.96 & 26.90 & 28.51 & 29.31 \\
\hline & SSIM & 0.74 & 0.64 & 0.79 & 0.81 \\
\hline \multirow{2}{*}{ 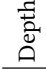 } & RMSE [m] & 0.21 & 0.32 & 0.23 & 0.20 \\
\hline & MAE [m] & 0.15 & 0.20 & 0.15 & 0.12 \\
\hline
\end{tabular}

Table 2. Comparison with other DOEs. We compare the average accuracy of the test sets of 145 HS-D images reconstructed with four different DOEs. Results are evaluated against the ground truth in the simulation. Our method outperforms three existing DOE designs in terms of spectral and depth accuracy. 

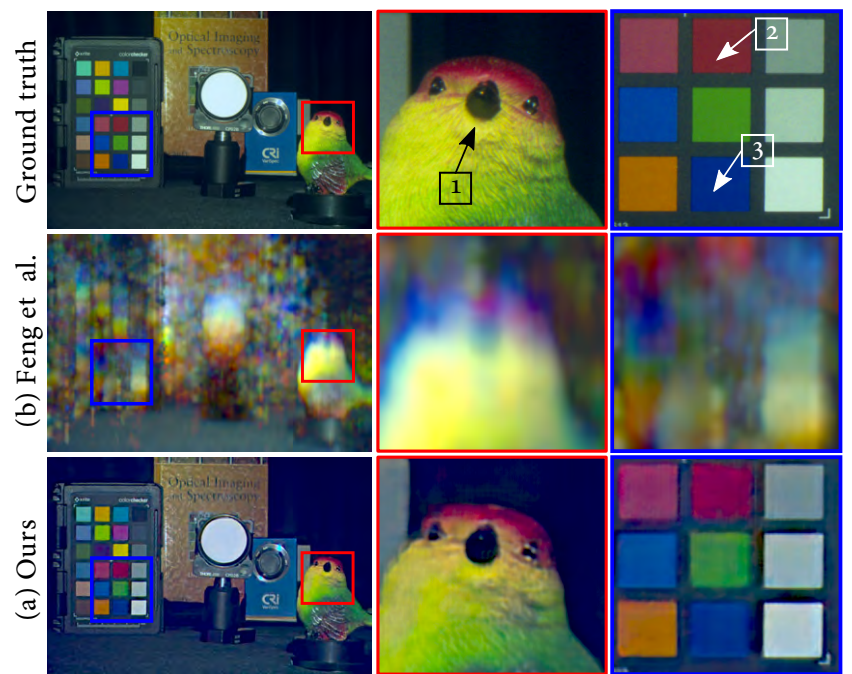

Reconstructed spectral image

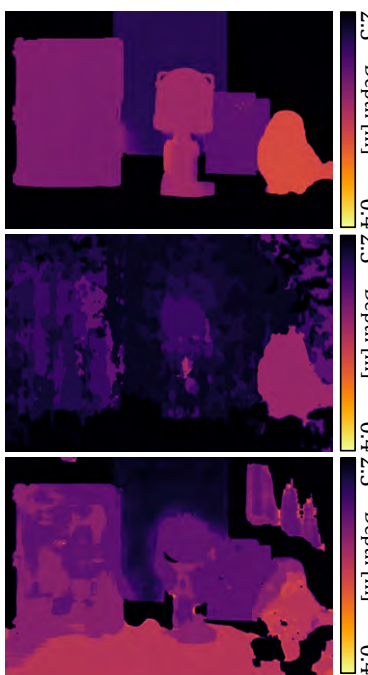

Depth map

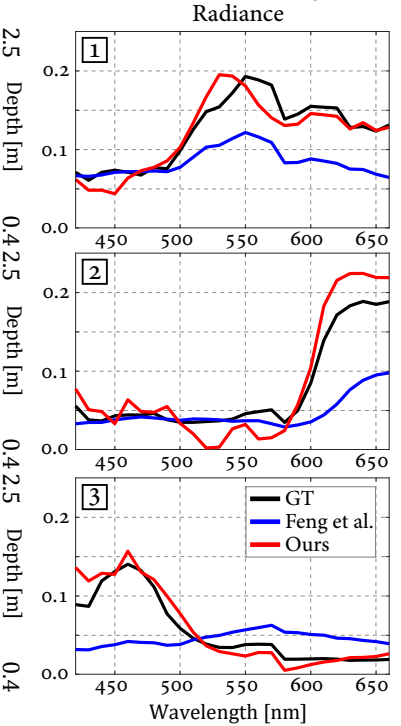

Fig. 9. Comparison with other HS-D imaging in a simulation with the ground truth. This figure presents an example of reconstructed radiance maps of scale-normalized spectral power distributions and depth maps. Existing single-shot HS-D imaging methods combine CASSI for spectral analysis with additional micro-lens array to obtain depth information [Feng et al. 2016]. With a learned DOE, our single-shot HS-D imaging provides better reconstruction both in terms of spectrum and depth.
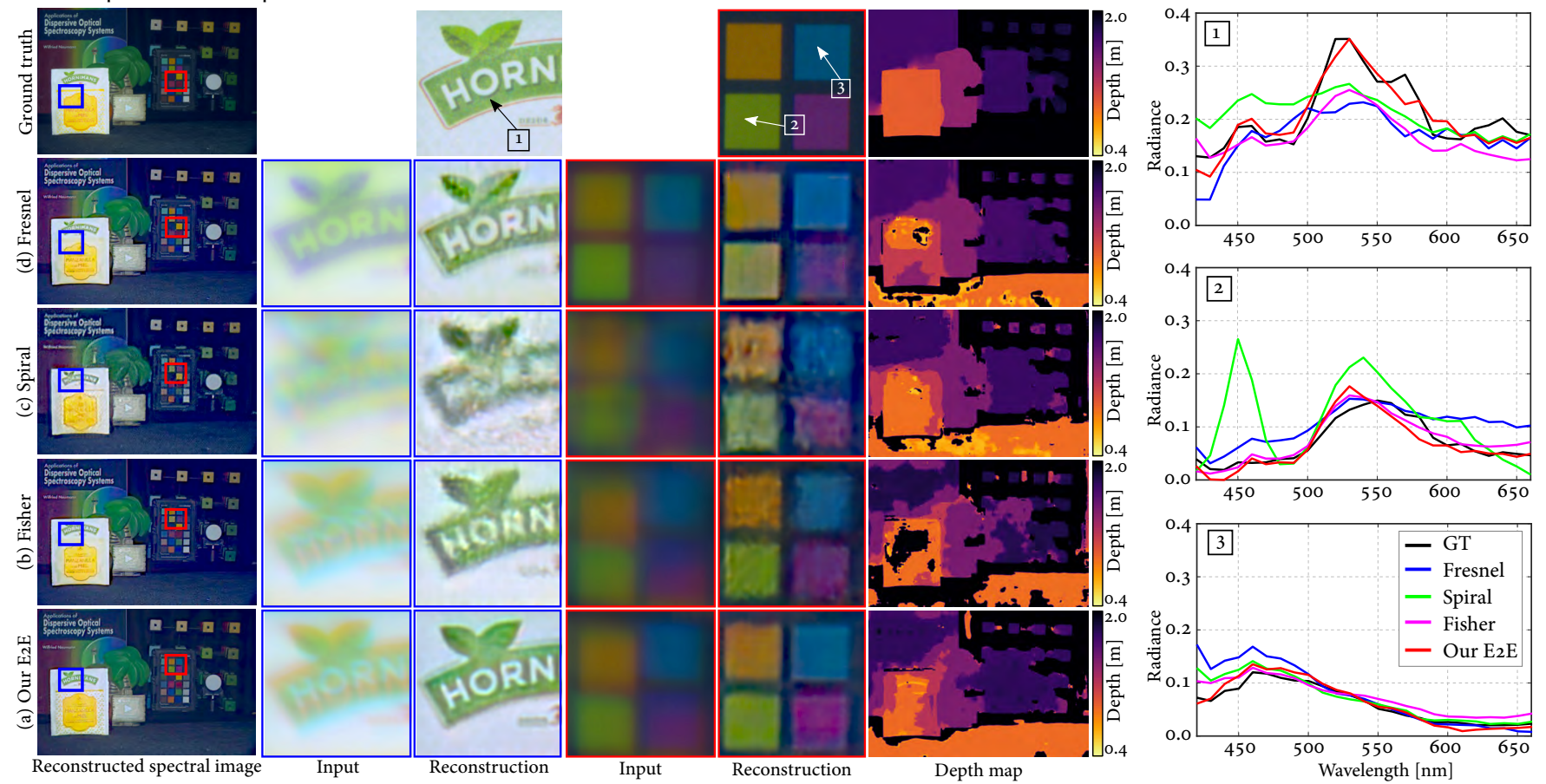

Fig. 10. Comparison with other DOEs in a simulation with the ground truth. Here we qualitatively compare the image quality and spectral accuracy of reconstructed hyperspectral images and the accuracy of depth maps. The Fresnel lens, spiral DOE, Fisher-based DOE are fixed when training reconstruction networks. In contrast, our end-to-end DOE is learned together with network parameters. The plots at the bottom present the scale-normalized radiance of the area marked by small squares. Our method outperforms all three DOEs in terms of spectral accuracy, image structure and depth accuracy.

while the spectral results are suboptimal (Figure 10(d)). The Fisherinformation DOE performs well in reconstructing both spectrum and depth information; however, the reconstructed hyperspectral images suffer from significant noise (Figure 10(b)). The performance of the spiral DOE is worse in terms of spectrum and depth reconstruction (Figure 10(c)). We hypothesize that an advanced network architecture, such as the unrolled network architecture used by Jeon et al. [2019], might be required to handle the complex patterns of the spiral PSF. In contrast, our end-to-end optimization learns 
not only DOE patterns but also network parameters for better representations of spectrum and depth information in natural image characteristics. Our method outperforms three state-of-the-art DOE methods overall (Figure 10(a)). In particular, our method is significantly better in terms of both spectral accuracy and preservation of image structures.

We evaluated each Cramer-Rao Lower Bound (CRLB) value (the lower value, the more discriminating power) of different DOEs with/without end-to-end optimization. Initial CRLBs of the fixed Fresnel/Spiral/Fisher DOEs are 2.73/1.89/1.36, respectively. After end-to-end optimization, they become 5.28/3.32/3.00, respectively. If we capture the spectrum and depth of a single point, the fixed Fisher DOE is the best performing DOE. However, it is not optimal for spectral and depth imaging where the scene is complex. The fixed DOEs cannot guarantee clear hyperspectral images and fails to recover its spectral data accurately. In contrast, our end-to-end DOE produces not only sharp but also accurate spectral images and depth maps.

Comparison with other hyperspectral imaging. Figure 11 compares our system (a) with two recent compact hyperspectral imaging systems: (b) a spiral DOE-based spectral imaging method [Jeon et al 2019] and (c) a prism-based spectral imaging method [Baek et al 2017] in a simulation with the ground truth. For Jeon et al. and ours, we use the half resolution of the test images in 1412-by-2120. For Baek et al., we reduce the resolution of the input image by one-eighth as their method takes about 45 minutes to process a 353 by-530 hyperspectral image. The table in Figure 11 presents average PSNR and SSIM metrics computed for hyperspectral cubes and corresponding luminance images on 145 test images. Our method is superior to other systems in terms of spectral accuracy, compared with the state-of-the-art systems. Jeon et al. present high-frequency spatial details without artifacts whereas its spectral accuracy is suboptimal. We speculate that this is due to them using a smaller aperture of $1 \mathrm{~mm}$ while we choose a larger aperture of $3 \mathrm{~mm}$ for simultaneous estimation of the spectrum and depth and better light efficiency. The spectral accuracy of these state-of-the-art methods is competitive; however, Baek et al. and Jeon et al. are limited to spectral estimation only. In contrast, our method can capture both spectrum and depth information from a single input image, while the spectral accuracy of our method is superior to the other existing snapshot spectral imaging methods.

Comparison with other depth imaging. We compared the performance of our approach (a) for depth-only imaging with two other DOE-based depth imaging methods: (b) Wu et al. [2019] and (c) Chang et al. [2019] in Figure 12. The experimental configurations of these three methods (Chang et al./Wu et al./ours) are all different including the effective pixel pitch (4.29/9.60/6.75 um), aperture diameter $(0.800 / 2.835 / 3.000 \mathrm{~mm})$, the network design (three different variants of U-net), the training dataset (real RGB-D dataset/synthetic RGB-D dataset/real HS-D dataset), and the camera response function. Therefore, we varied the design parameters of their DOEs only while fixing the other configuration parameters to be the same as ours. We implemented the phase shift of the thin lens for Chang et al. [2019] and used the DOE design provided by the authors for $\mathrm{Wu}$ et al. [2019]. The simulated PSFs for both are shown in Figure 12
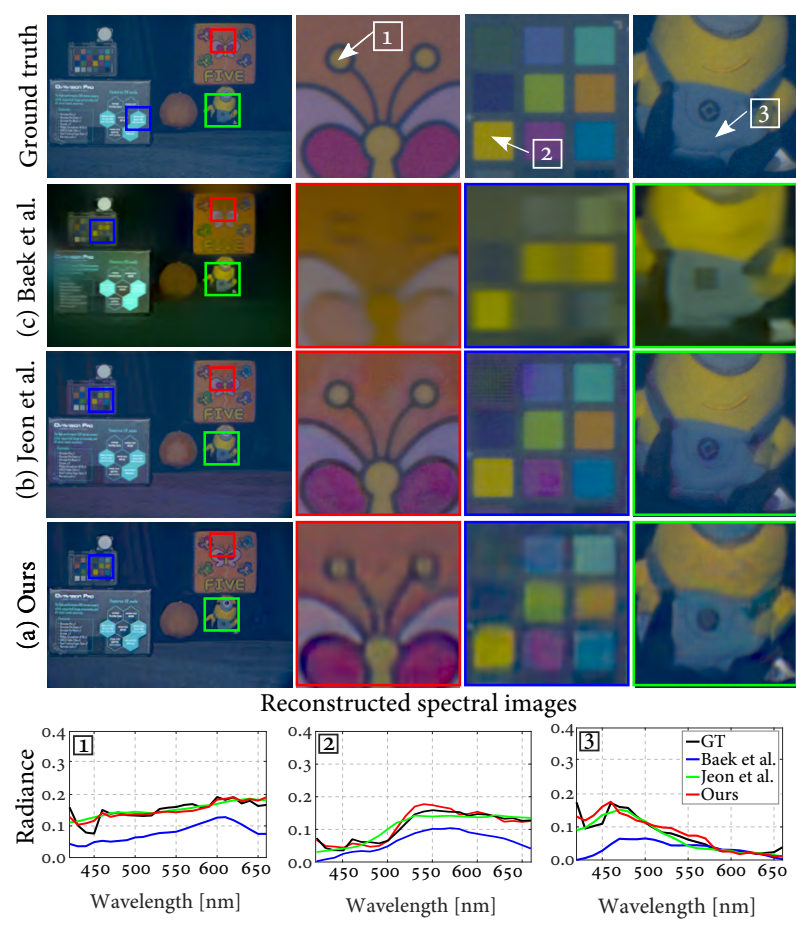

Reconstructed spectral images

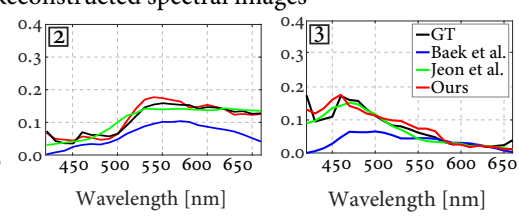

\begin{tabular}{c|r|r|r}
\hline HS imaging & Baek et al. & Jeon et al. & Ours \\
\hline \hline HS PSNR [dB] & 27.96 & 28.81 & $\mathbf{2 9 . 3 1}$ \\
HS SSIM & 0.75 & $\mathbf{0 . 8 1}$ & $\mathbf{0 . 8 1}$ \\
\hline Luminance PSNR [dB] & 28 & $\mathbf{4 0}$ & 32 \\
Luminance SSIM & 0.91 & $\mathbf{0 . 9 6}$ & 0.94 \\
\hline
\end{tabular}

Fig. 11. Comparison with state-of-the-art compact HS imaging methods in a simulation with the ground truth: the prism-based [Baek et al. 2017] and the spiral DOE-based hyperspectral system [Jeon et al. 2019]. We evaluate the spatial image quality and spectral accuracy by simulating the image formation model of each method. Our proposed method outperforms both approaches in spectral accuracy (PSNR and SSIM computed on the hyperspectral cube) while achieving second-best performance in terms of spatial structure (PSNR and SSIM computed on the luminance image of the hyperspectral cube). Note that our method acquires not only spectrum but also depth different from the both approaches.

and their shapes match those reported in the original works. The same U-net-based reconstruction network was used for all DOE designs, but trained differently with each DOE on our HS-D dataset. Note that the spectral decoder was deactivated in this experiment. As shown in Figure 12, our DOE for HS-D imaging yields superior results in terms of depth accuracy compared with the other DOE designs designed for only depth imaging.

Impact of DOE initialization. In recent studies on end-to-end optimization of optics, finding the best DOE is not a convex problem, and thus the DOE initialization [Chang and Wetzstein 2019; Wu et al. 2019] has a high impact on the performance. We therefore tested three different initial DOE designs for end-to-end HS-D imaging: the Fresnel lens, the spiral DOE [Jeon et al. 2019], and the Fisher-information-based DOE. Table 3 compares how much the end-to-end optimization process of optics improves the accuracy of 


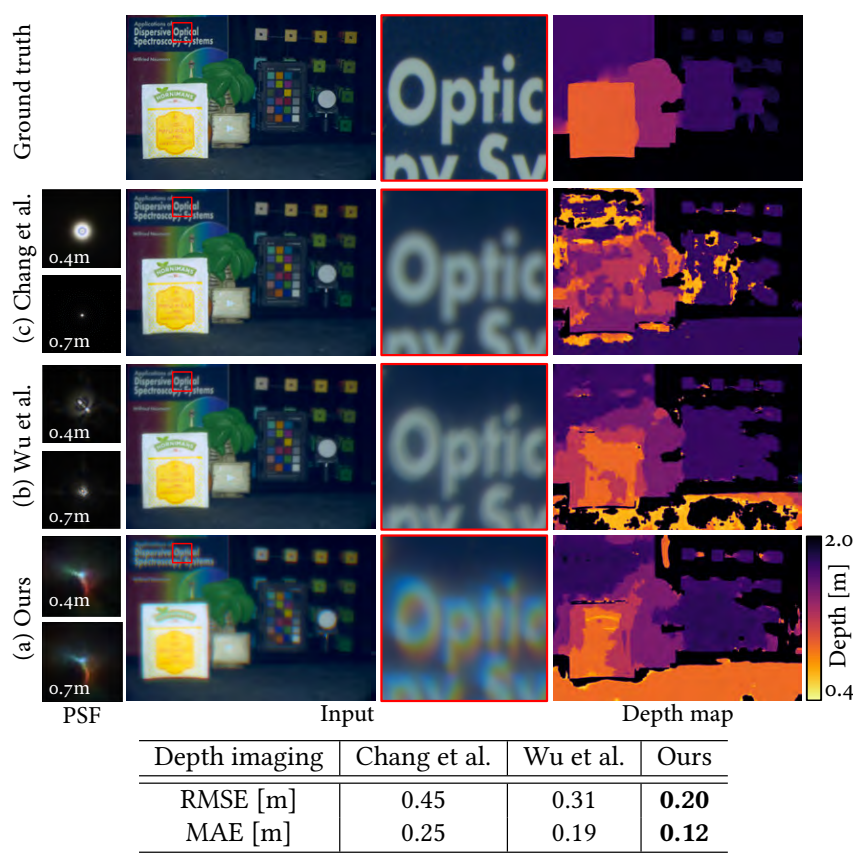

Fig. 12. Comparison with other PSF-based depth imaging in a simulation with the ground truth. We compare depth reconstruction results of our method with two state-of-the-art methods that estimate depth using DOEs [Chang and Wetzstein 2019; Wu et al. 2019]: the traditional thin lens and the depth-optimized Fisher-based DOE. Our learned DOE lets the network acquire depth representation from the scenes effectively, producing superior results in terms of depth accuracy.

reconstructed spectral and depth information for different initializations. Among the three candidates, we chose the Fisher-based initialization as it is superior to other initializations in terms of spectral and depth accuracy. Refer to Section 5 the supplemental document for more quantitative evaluation.

\begin{tabular}{|c|c|c|c|c|}
\hline \multicolumn{2}{|c|}{ Initialization } & Fresnel & Spiral & Fisher \\
\hline \multirow{2}{*}{ ڤัँ } & PSNR [dB] & 28.68 & 27.67 & 29.31 \\
\hline & SSIM & 0.78 & 0.75 & 0.81 \\
\hline \multirow{2}{*}{$\begin{array}{l}\text { 离 } \\
\text { ص̆ }\end{array}$} & RMSE [m] & 0.19 & 0.26 & 0.20 \\
\hline & MAE [m] & 0.12 & 0.18 & 0.12 \\
\hline
\end{tabular}

Table 3. Impact of initialization DOE designs in a simulation. We compared the impacts of the three different initializations for our end-to-end HSD imaging. The Fisher-initialized DOE optimization is superior to other initializations for spectral and depth reconstruction, and the Fresnel-lensinitialized optimization is the second best option.

Scene illumination. We synthetically evaluate our end-to-end HSD imaging under 29 different CIE standard illuminants by averaging the hyperspectral PSNR and the depth RMSE values of five different test scenes. Figure 13 shows the results. Our HS-D imaging estimates depth with high accuracy consistently under various illuminations, except for one LED illuminant with a sharp peak near the infrared wavelength (LED-RGB1). This illuminant is almost monochromatic (at the spectral resolution of our system), and hence the images lack the spectral cues needed to infer the depth with high accuracy. We note however, that the depth estimation works well for more

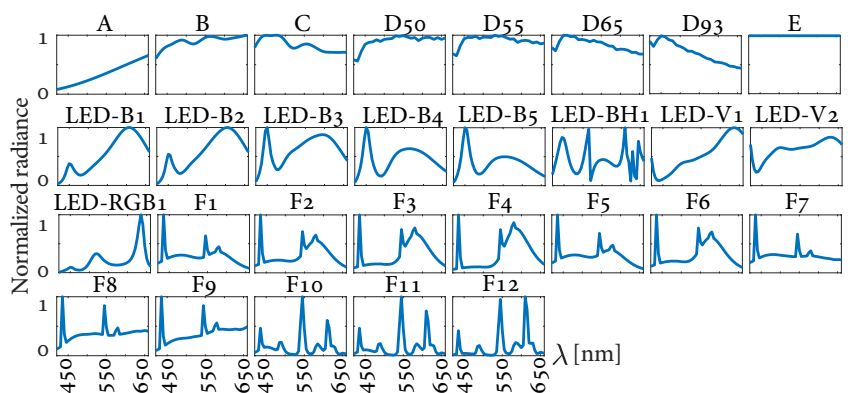

(a) Standard illuminants

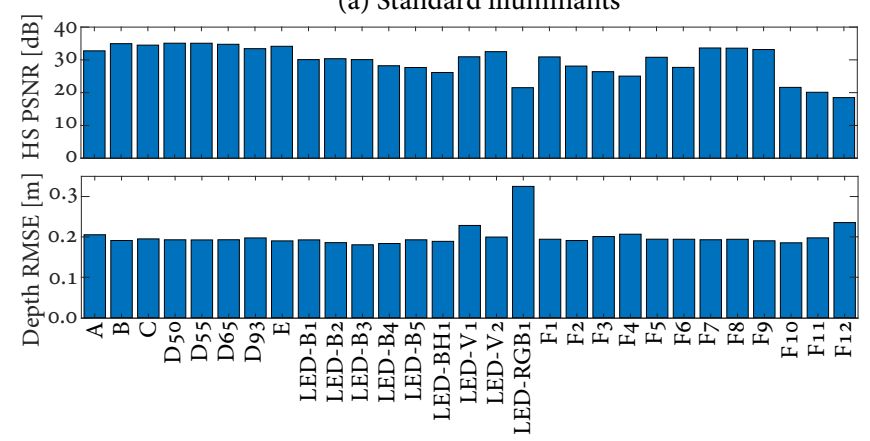

(b) Spectral and depth accuracy

Fig. 13. Reconstruction performance under different illuminants. (a) We evaluate our method on the HS-D test dataset augmented with 29 CIE standard illuminants. (b) Reconstruction accuracy of spectrum and depth is affected by the frequency of the illuminant as observed by degradation at fluorescent illuminants (F10-F12) and an LED illuminant (LED-RGB1).

natural types of illumination. Our method captures the spectral information with high accuracy under most illuminants in general. Under high-frequency illuminants, such as fluorescent F10, F11, F12, and LED-RGB1, our spectral reconstruction performs suboptimally due to the strong-peak illumination of fluorescent and LED light. We found that our end-to-end HS-D imaging performs robustly under sun, tungsten, general fluorescent, and general LED lights.

Spectral evaluation. In order to evaluate the spectral accuracy of our system, we compare the reconstructed radiance of 24 patches in the standard ColorChecker under the CIE D65 illuminant with the ground truth in the simulation. As shown in Figure 14, our results closely match the spectral power distributions of every patch in the ground truth data, although we intentionally excluded the ColorChecker images in the training process to avoid overfitting of the network parameters to this target. The mean RMSE of reflectance $(0.0-1.0)$ of all 24 patches is just 0.0478 .

Results with real prototype. Our compact prototype (described in Section 6) enables casual hyperspectral imaging of indoor and outdoor scenes. We captured five real-world scenes, as shown in Figures 1 and 15. These scenes are compared with the ground truth measured by a spectroradiometer and a laser distance meter. To reconstruct spectrum and depth information, we refined the reconstruction network parameters with the calibrated PSFs of our prototype (shown in Figure 8). Figure 15 shows the reconstructed hyperspectral images (visualized in sRGB) with spectral plots and 

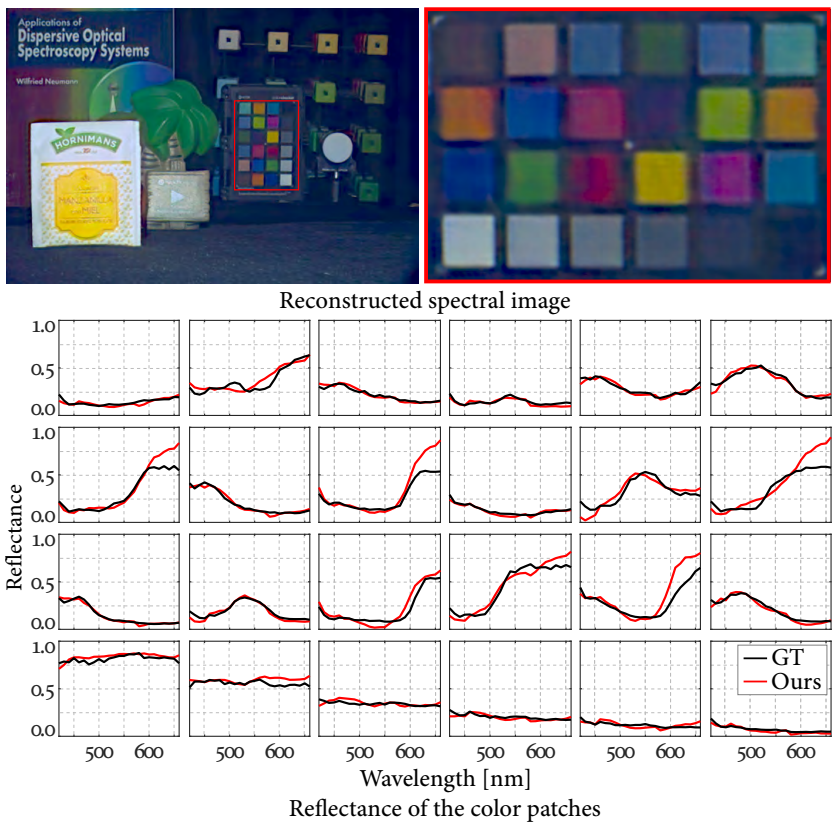

Fig. 14. Quantitative evaluation of our HS-D imaging with a ColorChecker in a simulation with the ground truth. Spectral plots of 24 patches in the ColorChecker present spectral reconstruction of our method with high accuracy.

depth maps. We compare our hyperspectral image measurements with measurements by the reference spectroradiometer and depth values with the reference laser distance measure.

Reconstruction refinement. As described in Section 6.3, there is a gap between the synthetically optimized DOE and the fabricated DOE, producing the hazy artifacts that degrade the performance of the real prototype. Our refinement process enhances the system performance by updating the parameters in the reconstruction network with respect to the real DOE. Figure 16 compares reconstruction results of spectral and depth images. In particular, the accuracy of depth information has been improved more significantly.

In addition, we evaluate the spatial resolution of our real-prototype results in terms of modulation transfer function (MTF) by capturing a spatial-resolution target as shown in Figure 17. These two input and output images are converted to luminance to compute MTFs. Qualitative and quantitative results show that the spatial resolution is improved by our reconstruction process for the real-DOE prototype.

\section{DISCUSSION}

Spatial variance of PSF. The computational burden of simulating our HS-D image formation prohibits additional computational cost of simulating spatially-varying PSFs. To validate our assumption that the spatial variation of PSF is insignificant, as shown in the previous PSF-engineering studies [Chang and Wetzstein 2019; Jeon et al. 2019; Wu et al. 2019], we compared our PSFs at the orthogonal incident angle and the 8-degree slanted angle, which corresponding to $\sim 60 \%$ of the vertical FOV (27 degrees). The SSIM between the
PSFs is 0.9988 indicating insignificant spatial variance because of the large $f$-number of 16 .

Spectral-depth tradeoff. Since we aim to estimate both spectrum and depth, the DOE and reconstruction network could be optimized favorably for one of them. While adjusting the weights of the loss function in Equation (7) can balance this, it would be interesting to develop a method for handling this tradeoff in a fairer manner. Also, we observed that there are similarly shaped PSFs in the spectrum and depth slices of the optimized PSF. Even though we alleviate this with the reconstruction network by learning spatio-spectral priors, the ambiguity in the PSF is still challenging to resolve perfectly. To improve the reconstruction quality for spectrum and depth information, it would be worthwhile to optically resolve this ambiguity using multiple DOEs or other optical elements in future work.

Training strategy. The optimization problem for end-to-end optics includes many non-convex optimization problems and thus the initialization is critical and the training strategy of both optics and neural network is also important. In this work, we followed the existing conventions for the DOE initialization and network training in recent studies [Chang and Wetzstein 2019; Wu et al. 2019]. Developing better training strategies for end-to-end optimization would be an interesting avenue of future work. Also, employing more advanced reconstruction schemes inspired by traditional optimization methods would be helpful to make the reconstruction interpretable in terms of end-to-end optics, even though it was not feasible in this work because of the demanding GPU memory for the problem of HS-D simulation and reconstruction.

Textureless and saturated regions. Our method falls in the regime of PSF engineering approaches, which fundamentally depend on texture information, similar to stereo imaging or depth-from-defocus imaging. This limits the accuracy of the reconstruction quality on texture-less or saturated surfaces as shown in the various results. In future work, it would be interesting to simultaneously estimate reconstruction confidence maps in addition to spectrum and depth, and then propagate the highly confident reconstruction to the regions with low confidence. Figure 18 shows a failure case of reconstruction on the saturated pixel regions.

Spectral range. In principle, our proposed method can be extended to a wider spectral range (e.g., infrared wavelengths). However, our target spectral range is limited to visible spectrum (420 to $660 \mathrm{~nm}$ ) by the camera response function of a DSLR camera. It would be an interesting future work of extending our method to different spectral ranges.

\section{CONCLUSION}

We have presented a novel end-to-end hyperspectral-depth imaging system that consists of a learned DOE and a conventional DSLR camera, enabling compact and portable hyperspectral 3D imaging. In addition, we have provided a unique hyperspectral-depth dataset of indoor scenes that enables us to train the optics and neural network for simultaneously capturing hyperspectral-depth scene information, using a single RGB image, with a learned DOE. The learned DOE is fabricated with a grayscale lithography process and inserted 

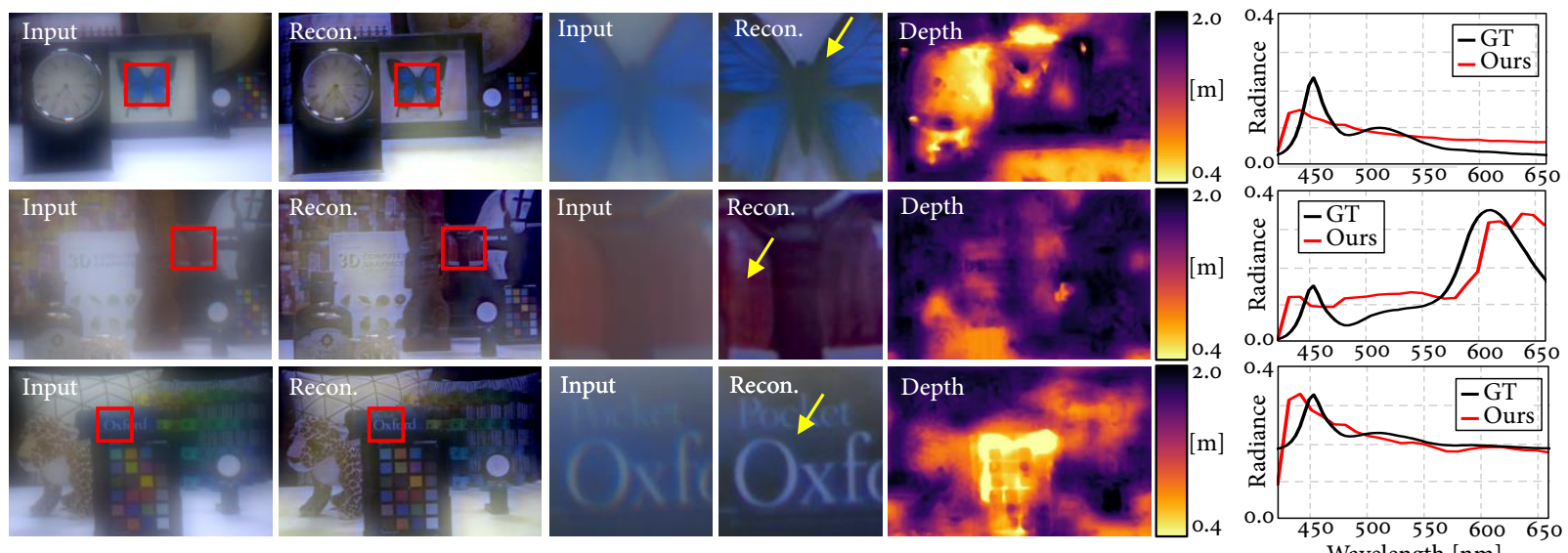

Fig. 15. Reconstructed hyperspectral-depth images of real-world, casual scenes. We captured these scenes with our prototype and compare the normalized radiance of resulting HS-D data with the ground truth measured by a spectroradiometer at points indicated by yellow arrows.

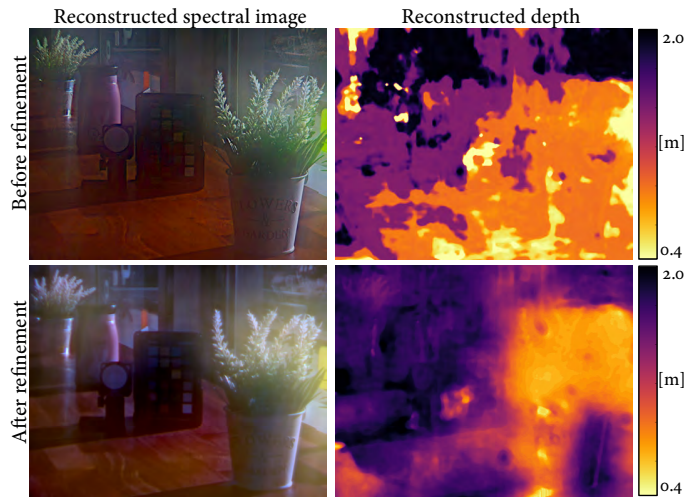

Fig. 16. Comparison of reconstruction results with/without the additional refinement process for the real-DOE prototype. The additional refinement of the reconstruction network improves depth accuracy in particular.

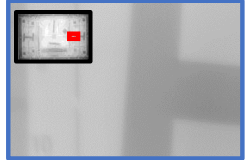

Input

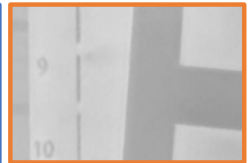

Output

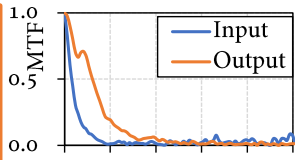

O.0 0.10 .2 Frequency (cycle 0.4 pixel) 0.5
Fig. 17. Spatial resolution analysis of input and output spectral images of our real prototype. These two images are converted to luminance to compute MTFs. The MTF of output is clearly improved by our reconstruction network.
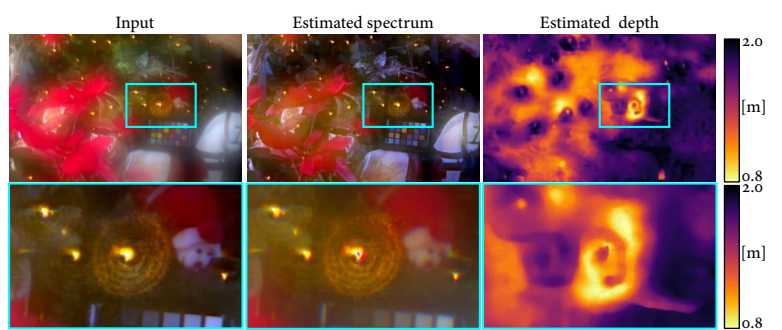

Fig. 18. On regions where pixels are saturated by specular highlights, our method results in reconstruction artifacts in both the spectrum and depth map.

into our portable HS-D camera prototype. We have demonstrated results of robustly capturing HS-D information on various natural scenes. The spatial and spectral accuracy of our technique is superior to previous approaches while our technique simultaneously captures hyperspectral-depth scene information.

\section{REFERENCES}

Telmo Adao, Jonas Hruska, Luis Padua, Jose Bessa, Emanuel Peres, Raul Morais, and Joaquim Joao Sousa. 2017. Hyperspectral imaging: A review on UAV-based sensors, data processing and applications for agriculture and forestry. Remote Sensing 9, 11 (2017), 1110.

Seung-Hwan Baek, Incheol Kim, Diego Gutierrez, and Min H Kim. 2017. Compact single-shot hyperspectral imaging using a prism. ACM Transactions on Graphics (TOG) 36, 6 (2017), 217.

David J. Brady. 2009. Optical Imaging and Spectroscopy. John Wiley \& Sons, Inc.

X Briottet, Y Boucher, A Dimmeler, A Malaplate, A Cini, Marco Diani, HHPT Bekman, P Schwering, T Skauli, I Kasen, et al. 2006. Military applications of hyperspectral imagery. In Targets and backgrounds XII: Characterization and representation, Vol. 6239. International Society for Optics and Photonics, 62390B.

Vladimir Bychkovsky, Sylvain Paris, Eric Chan, and Frédo Durand. 2011. Learning Photographic Global Tonal Adjustment with a Database of Input / Output Image Pairs. In The Twenty-Fourth IEEE Conference on Computer Vision and Pattern Recognition.

Ayan Chakrabarti. 2016. Learning sensor multiplexing design through back-propagation In Advances in Neural Information Processing Systems. 3081-3089.

Ayan Chakrabarti and Todd Zickler. 2011. Statistics of real-world hyperspectral images. In Computer Vision and Pattern Recognition (CVPR), 2011 IEEE Conference on. IEEE, 193-200.

Julie Chang, Vincent Sitzmann, Xiong Dun, Wolfgang Heidrich, and Gordon Wetzstein. 2018. Hybrid optical-electronic convolutional neural networks with optimized diffractive optics for image classification. Scientific reports 8, 1 (2018), 12324.

Julie Chang and Gordon Wetzstein. 2019. Deep Optics for Monocular Depth Estimation and 3D Object Detection. In IEEE International Conference on Computer Vision (ICCV).

Inchang Choi, Daniel S. Jeon, Giljoo Nam, Diego Gutierrez, and Min H. Kim. 2017. HighQuality Hyperspectral Reconstruction Using a Spectral Prior. ACM Transactions on Graphics (Proc. SIGGRAPH Asia 2017) 36, 6 (2017).

Laura M Dale, André Thewis, Christelle Boudry, Ioan Rotar, Pierre Dardenne, Vincent Baeten, and Juan A Fernández Pierna. 2013. Hyperspectral imaging applications in agriculture and agro-food product quality and safety control: a review. Applied Spectroscopy Reviews 48, 2 (2013), 142-159.

Gerwin Damberg, James Gregson, and Wolfgang Heidrich. 2016. High brightness HDR projection using dynamic freeform lensing. ACM Transactions on Graphics (TOG) 35,3 (2016), 24

Weiyi Feng, Hoover Rueda, Chen Fu, Gonzalo R Arce, Weiji He, and Qian Chen. 2016. 3D compressive spectral integral imaging. Optics express 24, 22 (2016), 24859-24871.

Joseph W Goodman. 2005. Introduction to Fourier optics. Roberts and Company Publishers.

Harel Haim, Shay Elmalem, Raja Giryes, Alex M Bronstein, and Emanuel Marom. 2018. Depth estimation from a single image using deep learned phase coded mask. IEEE Transactions on Computational Imaging 4, 3 (2018), 298-310.

Shuya Ito, Koichi Ito, Takafumi Aoki, and Masaru Tsuchida. 2016. A 3D Reconstruction Method with Color Reproduction from Multi-band and Multi-view Images. In Asian 
Conference on Computer Vision. Springer, 236-247.

Daniel S Jeon, Seung-Hwan Baek, Shinyoung Yi, Qiang Fu, Xiong Dun, Wolfgang Heidrich, and Min H Kim. 2019. Compact snapshot hyperspectral imaging with diffracted rotation. ACM Transactions on Graphics (TOG) 38, 4 (2019), 117.

Daniel S Jeon, Inchang Choi, and Min H Kim. 2016. Multisampling Compressive Video Spectroscopy. Computer Graphics Forum 35, 2 (2016), 467-477.

William R Johnson, Daniel W Wilson, Wolfgang Fink, Mark Humayun, and Greg Bearman. 2007. Snapshot hyperspectral imaging in ophthalmology. Fournal of biomedical optics 12, 1 (2007), 014036-014036.

Min H Kim, Todd Alan Harvey, David S Kittle, Holly Rushmeier, Julie Dorsey, Richard O Prum, and David J Brady. 2012. 3D imaging spectroscopy for measuring hyperspectral patterns on solid objects. ACM Transactions on Graphics 31, 4 (2012), 38

Masahiro Kitahara, Takahiro Okabe, Christian Fuchs, and Hendrik PA Lensch. 2015 Simultaneous Estimation of Spectral Reflectance and Normal from a Small Number of Images.. In VISAPP (1). 303-313.

Douglas Lanman and Gabriel Taubin. 2009. Build your own 3D scanner: optical triangulation for beginners. In ACM SIGGRAPH ASIA 2009 Courses. ACM, 2.

Guolan Lu and Baowei Fei. 2014. Medical hyperspectral imaging: a review. fournal of biomedical optics 19, 1 (2014), 010901.

C. Metzler, H. Ikoma, Y. Peng, and G. Wetzstein. 2020. Deep Optics for Single-shot High-dynamic-range Imaging. In Proc. CVPR.

Roope Näsi, Eija Honkavaara, Päivi Lyytikäinen-Saarenmaa, Minna Blomqvist, Paula Litkey, Teemu Hakala, Niko Viljanen, Tuula Kantola, Topi Tanhuanpää, and Markus Holopainen. 2015. Using UAV-based photogrammetry and hyperspectral imaging for mapping bark beetle damage at tree-level. Remote Sensing 7, 11 (2015), 15467-15493.

Elias Nehme, Daniel Freedman, Racheli Gordon, Boris Ferdman, Lucien E Weiss, Onit Alalouf, Reut Orange, Tomer Michaeli, and Yoav Shechtman. 2019. DeepSTORM3D dense three dimensional localization microscopy and point spread function design by deep learning. arXiv preprint arXiv:1906.09957v2 (2019).

Keisuke Ozawa, Imari Sato, and Masahiro Yamaguchi. 2017. Hyperspectral photometric stereo for a single capture. JOSA A 34, 3 (2017), 384-394.

Yifan Peng, Oilin Sun, Xiong Dun, Gordon Wetzstein, Wolfgang Heidrich, and Felix Heide. 2019. Learned large field-of-view imaging with thin-plate optics. $A C M$ Transactions on Graphics (TOG) 38, 6 (2019), 219.

Olaf Ronneberger, Philipp Fischer, and Thomas Brox. 2015. U-net: Convolutional networks for biomedical image segmentation. In International Conference on Medical image computing and computer-assisted intervention. Springer, 234-241.

Hoover Rueda-Chacon, Juan F Florez, Daniel Leo Lau, and Gonzalo R Arce. 2019. Snapshot Compressive ToF+ Spectral Imaging via Optimized Color-Coded Apertures. IEEE transactions on pattern analysis and machine intelligence (2019).

Yoav Shechtman, Steffen J Sahl, Adam S Backer, and WE Moerner. 2014. Optimal point spread function design for 3D imaging. Physical review letters 113, 13 (2014), 133902.

Nathan Silberman, Derek Hoiem, Pushmeet Kohli, and Rob Fergus. 2012. Indoor segmentation and support inference from rgbd images. In European Conference on Computer Vision. Springer, 746-760.

Vincent Sitzmann, Steven Diamond, Yifan Peng, Xiong Dun, Stephen Boyd, Wolfgang Heidrich, Felix Heide, and Gordon Wetzstein. 2018. End-to-end optimization of optics and image processing for achromatic extended depth of field and super-resolution imaging. ACM Transactions on Graphics (TOG) 37, 4 (2018), 114.

Shuran Song, Samuel P Lichtenberg, and Jianxiong Xiao. 2015. Sun rgb-d: A rgb-d scene understanding benchmark suite. In Proceedings of the IEEE conference on computer vision and pattern recognition. 567-576.

Qilin Sun, Ethan Tseng, Qiang Fu, Wolfgang Heidrich, and Felix Heide. 2020a. Learning Rank-1 Diffractive Optics for Single-shot High Dynamic Range Imaging. IEEE CVPR (2020).

Quilin Sun, Jian Zhang, Xiong Dun, Bernard Ghanem, Yifan peng, and Wolfgang Heidrich. 2020b. End-to-End Learned, Optically Coded Super-resolution SPAD Camera. ACM Transactions on Graphics (TOG) 39 (2020).

Jonas Uhrig, Nick Schneider, Lukas Schneider, Uwe Franke, Thomas Brox, and Andreas Geiger. 2017. Sparsity Invariant CNNs. In International Conference on 3D Vision $(3 D V)$.

Ashwin Wagadarikar, Renu John, Rebecca Willett, and David Brady. 2008. Single disperser design for coded aperture snapshot spectral imaging. Applied optics 47, 10 (2008), B44-B51.

Lizhi Wang, Zhiwei Xiong, Guangming Shi, Wenjun Zeng, and Feng Wu. 2016. Simultaneous Depth and Spectral Imaging with A Cross-Modal Stereo System. IEEE Transactions on Circuits and Systems for Video Technology (2016).

Lizhi Wang, Tao Zhang, Ying Fu, and Hua Huang. 2019. HyperReconNet: Joint Coded Aperture Optimization and Image Reconstruction for Compressive Hyperspectral Imaging. IEEE Transactions on Image Processing 28, 5 (May 2019), 2257-2270. https: //doi.org/10.1109/TIP.2018.2884076

Jiamin Wu, Bo Xiong, Xing Lin, Jijun He, Jinli Suo, and Qionghai Dai. 2016. Snapshot hyperspectral volumetric microscopy. Scientific Reports 6 (2016), 24624.

Yicheng Wu, Vivek Boominathan, Huaijin Chen, Aswin Sankaranarayanan, and Ashok Veeraraghavan. 2019. PhaseCam3D-Learning Phase Masks for Passive Single View Depth Estimation. In IEEE International Conference on Computational Photography
(ICCP). 1-12.

Younan Xia and George M Whitesides. 1998. Soft lithography. Annual review of materials science 28, 1 (1998), 153-184.

Fumihito Yasuma, Tomoo Mitsunaga, Daisuke Iso, and Shree K Nayar. 2010. Generalized assorted pixel camera: postcapture control of resolution, dynamic range, and spectrum. IEEE Transactions on Image Processing 19, 9 (2010), 2241-2253.

Ali Zia, Jie Liang, Jun Zhou, and Yongsheng Gao. 2015. 3D reconstruction from hyperspectral images. In 2015 IEEE Winter Conference on Applications of Computer Vision. IEEE, 318-325. 